\begin{tabular}{|c|l|}
\hline Title & Numerical evidence for the bottleneck frequency of quasidiffusive acoustic phonons \\
\hline Author(s) & Tamura, Shin-ichiro \\
\hline Citation & $\begin{array}{l}\text { PHY SICAL REVIEW B, 56(21), 13630-13633 } \\
\text { https://doi.org/L0.1103/PhysRevB.56.13630 }\end{array}$ \\
\hline Issue Date & 1997 \\
\hline Doc URL & http://hdl.handle.net/2115/5918 \\
\hline Rights & Copyright $\odot 1997$ A merican Physical Society \\
\hline Type & article \\
\hline File Information & PRB56-21.pdf \\
\hline
\end{tabular}

Instructions for use 


\title{
Numerical evidence for the bottleneck frequency of quasidiffusive acoustic phonons
}

\author{
Shin-ichiro Tamura \\ Department of Applied Physics, Hokkaido University, Sapporo 060, Japan
}

(Received 25 July 1997)

\begin{abstract}
A kinetic equation on quasidiffusion of phonons was recently analyzed by Esipov and he predicted the existence of a bottleneck frequency $\left(\nu_{\mathrm{BN}}\right)$ which separates the phonons decaying from those diffusing to a detector. We have solved numerically the kinetic equation and obtained the temporal evolution of phonon concentration excited at the center of a spherical sample. We have also performed Monte Carlo simulations of phonon propagation in the same geometry. At a time much later than the ballistic arrival time of phonons, both sets of results exhibit a sharp peak in the phonon concentration around the predicted $\nu_{\mathrm{BN}}$. With Monte Carlo simulations we have also confirmed the same relaxation rate for the phonons of frequencies $\nu<\nu_{\mathrm{BN}}$. [S0163-1829(97)01645-7]
\end{abstract}

Propagation of high-frequency acoustic phonons in nonmetallic crystals at low temperatures is governed by the (1) focusing effect due to the anisotropy of the lattice, ${ }^{1,2}$ (2) elastic scattering due to foreign and isotopic impurities, ${ }^{3}$ and (3) anharmonic decay via three-phonon interaction. ${ }^{4-6}$ The propagation in the regime where the latter two effects are dominant is called quasidiffusion. ${ }^{7-10}$ Because of a highlyfrequency-dependent decay rate of phonons $\tau_{A}^{-1}=A \nu^{5}$ ( $A$ is a constant depending on phonon polarization and $\nu$ is the frequency), there exists a characteristic frequency $\nu(t)$ at an elapsed time $t$ determined by $\tau_{A}(\nu)=t$. This frequency gives the length $l$ of space expansion at a time $t$ as $l \sim v\left[\tau_{A}(\nu) \tau_{I}(\nu)\right]^{1 / 2} \sim t^{9 / 10}$, where $v$ is the Debye velocity and $\tau_{I}^{-1}=B \nu^{4}$ ( $B$ is a constant) is the elastic scattering rate. $^{7,8}$ Thus the average phonon distribution in a sample spreads more slowly than in ballistic propagation but faster than in normal diffusion.

More recent observations show that the characteristic behavior of quasidiffusive phonons appears in the time trace of the detected phonon signal as an exponentially decaying tail at a time much later than the ballistic time of flight $t_{b}$ through the sample. ${ }^{11-13}$ Experimentally, this exponential behavior has been observed for several semiconducting samples of slab geometry such as silicon, germanium, and GaAs with a photoexcitation technique at a low input power level. ${ }^{13}$ It is critical for observing the exponential tails to remove liquid helium from the surface where the phonons are excited. Otherwise, the high-frequency phonons excited at the sample surface are lost into the helium bath and the quasidiffusive tail originating from these phonons is hardly observed. The quasidiffusion is also important in analyzing the phonon signal produced by high-energy particles in a crystal. $^{14-18}$

The applicability of a quasidiffusive model is seen by comparing the experimental heat pulses with Monte Carlo simulations. ${ }^{19-21}$ Originally a simple one-branch model was proposed, where three-phonon polarizations were approximated by a single mode with the Debye velocity. ${ }^{19}$ The Monte Carlo simulations based on this model can reproduce the exponential tail in the time-of-flight spectrum of phonons arriving at a detector for $t \gg t_{b}$ though they fail to account for the shape of the phonon signal arriving at $t \simeq t_{b} \cdot{ }^{20}$ In this sense the one-branch model is useful in discussing the behavior of phonons in the time region $t \gg t_{b}$. Simulations with a more sophisticated three-branch model taking account of focusing effects have also been done and they reproduce the shape of observed time traces well even for $t \simeq t_{b} \cdot{ }^{20,21}$

The kinetic equations describing the quasidiffusion of phonons have been proposed and analyzed by several authors. $^{7,8,19,22}$ Specifically, Esipov has recently studied the kinetic equation in the framework of a one-branch, isotropic model and found a novel result. ${ }^{22}$ He claims that the equation governing the phonon concentration $N(\nu, t)$ involves a characteristic frequency (called the bottleneck frequency $\nu_{\mathrm{BN}}$ ) which separates the phonons into two groups; i.e., the phonons with $\nu<\nu_{\mathrm{BN}}$ have the same relaxation time determined by $\nu_{\mathrm{BN}}$ but those with $\nu>\nu_{\mathrm{BN}}$ decay with time constants depending on their frequency. However, it is still not obvious if the existence of $\nu_{\mathrm{BN}}$ has a clearly visible influence on the solution $N(\nu, t)$ of the kinetic equation.

The purpose of the present work is to study the validity of the kinetic equation for quasidiffusion and to give explicit evidence for the presence of $\nu_{\mathrm{BN}}$. This is done by numerically solving the equation studied by Esipov and obtaining the time evolution of the phonon concentration. We also carry out Monte Carlo simulations for phonon propagation in the same sample geometry. As we shall see below, the results of both studies explicitly suggest the existence of a bottleneck frequency in the quasidiffusive regime. More specifically, at $t \gg t_{b}$ the numerical solution for the frequency distribution of phonons inside a sample exhibits a sharp peak at the predicted bottleneck frequency $\nu_{\mathrm{BN}}$. This distribution gives good agreement with the phonon concentration obtained by the Monte Carlo simulation if the initial phonon frequency is high enough. Also the simulations reveal that the tails of the detected phonon signals for $\nu<\nu_{\mathrm{BN}}$ decay with the same relaxation rate as predicted by Esipov.

We start with the kinetic equation that Esipov studied. ${ }^{22}$ Neglecting the upconversion of phonons, the kinetic equation governing the propagation of phonons in the one-branch isotropic model is written 


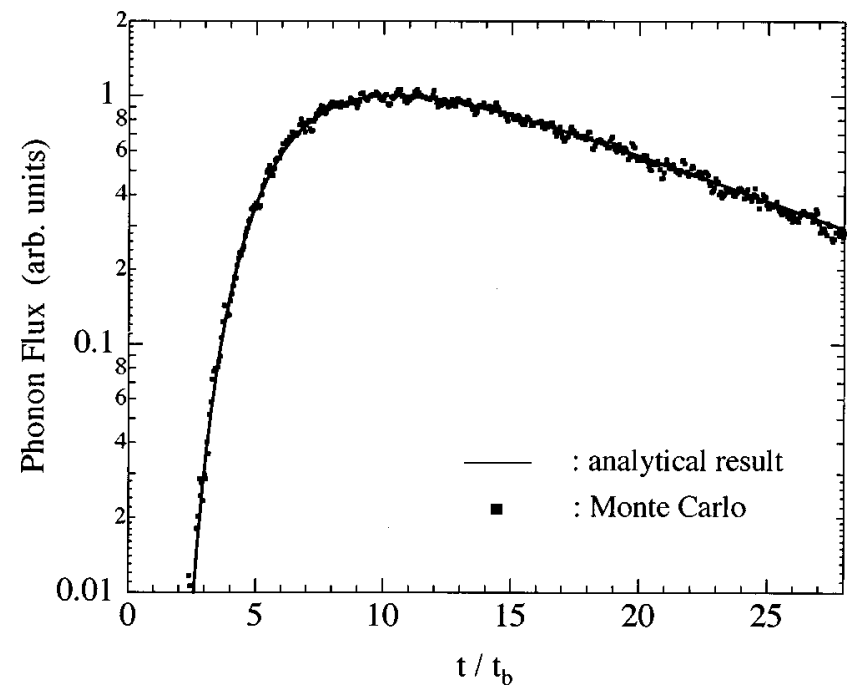

FIG. 1. Flux versus arrival time of purely diffusive phonons in silicon sphere of radius $R=5.5 \mathrm{~mm}$. Phonons of $2 \mathrm{THz}$ frequency are assumed to be generated at the center of the sphere at $t=0$. Dots are the Monte Carlo results and the solid line is the analytical result derived from Eq. (2).

$$
\begin{gathered}
{\left[\frac{\partial}{\partial t}-D(\nu) \nabla^{2}+\frac{1}{\tau_{A}(\nu)}\right] N(\nu, \mathbf{r}, t)} \\
=\int_{\nu}^{\infty} d \nu^{\prime} N\left(\nu^{\prime}, \mathbf{r}, t\right) \Gamma\left(\nu^{\prime}, \nu\right),
\end{gathered}
$$

where $N$ is the phonon concentration, $D(\nu)=\frac{1}{3} v^{2} \tau_{I}(\nu)$ is the averaged diffusivity, and $\Gamma\left(\nu^{\prime}, \nu\right)$ describes the probability of a phonon of frequency $\nu^{\prime}$ producing a phonon of frequency $\nu$ in a decay via a three-phonon process. The kernel $\Gamma\left(\nu^{\prime}, \nu\right)$ is assumed as $\Gamma\left(\nu^{\prime}, \nu\right)=\left[\tau_{A}\left(\nu^{\prime}\right)\right]^{-1} P\left(\nu^{\prime}, \nu\right)$ with $P\left(\nu^{\prime}, \nu\right)=60 \nu^{2}\left(\nu^{\prime}-\nu\right)^{2} /\left(\nu^{\prime}\right)^{5}$. This form of $P$ satisfying $\int_{0}^{\nu_{0}} \nu P\left(\nu_{0}, \nu\right) d \nu=\nu_{0}$ was originally introduced by Maris. ${ }^{19}$

First we consider the case of pure diffusion by neglecting the right-hand side (RHS) of Eq. (1) and also the term proportional to $\tau_{A}^{-1}$. Suppose that the phonons are excited at $t=0$ at the center of a spherical sample of radius $R$ and with the boundary condition $N(\nu, R, t)=0$. This boundary condition implies that the surface of the sphere is covered with a perfect absorber of phonons. The solution of the diffusion equation is then

$$
N(\nu, r, t)=N_{0} \sum_{n=1}^{\infty} \frac{n}{r} \sin \frac{n \pi r}{R} \exp \left[-n^{2} \pi^{2} D(\nu) t / R^{2}\right],
$$

where $N_{0}$ is the initial number of phonons. In order to check our Monte Carlo code we compare in Fig. 1 the flux $\left.\nabla_{r} N\right|_{r=R}$ derived from the solution (2) and the corresponding results of the Monte Carlo simulations for the phonon propagation in the presence of elastic scattering $\tau_{I}^{-1}=B \nu^{4}$ with $B=2.43 \times 10^{-42} \mathrm{~s}^{3}$, valid for silicon. The details of the Monte Carlo simulations are described in Ref. 20. As expected both results coincide very well. An important observation is that in the sum of Eq. (2), the first term $(n=1)$ gives the dominant contribution at large $t$. Thus Esipov has
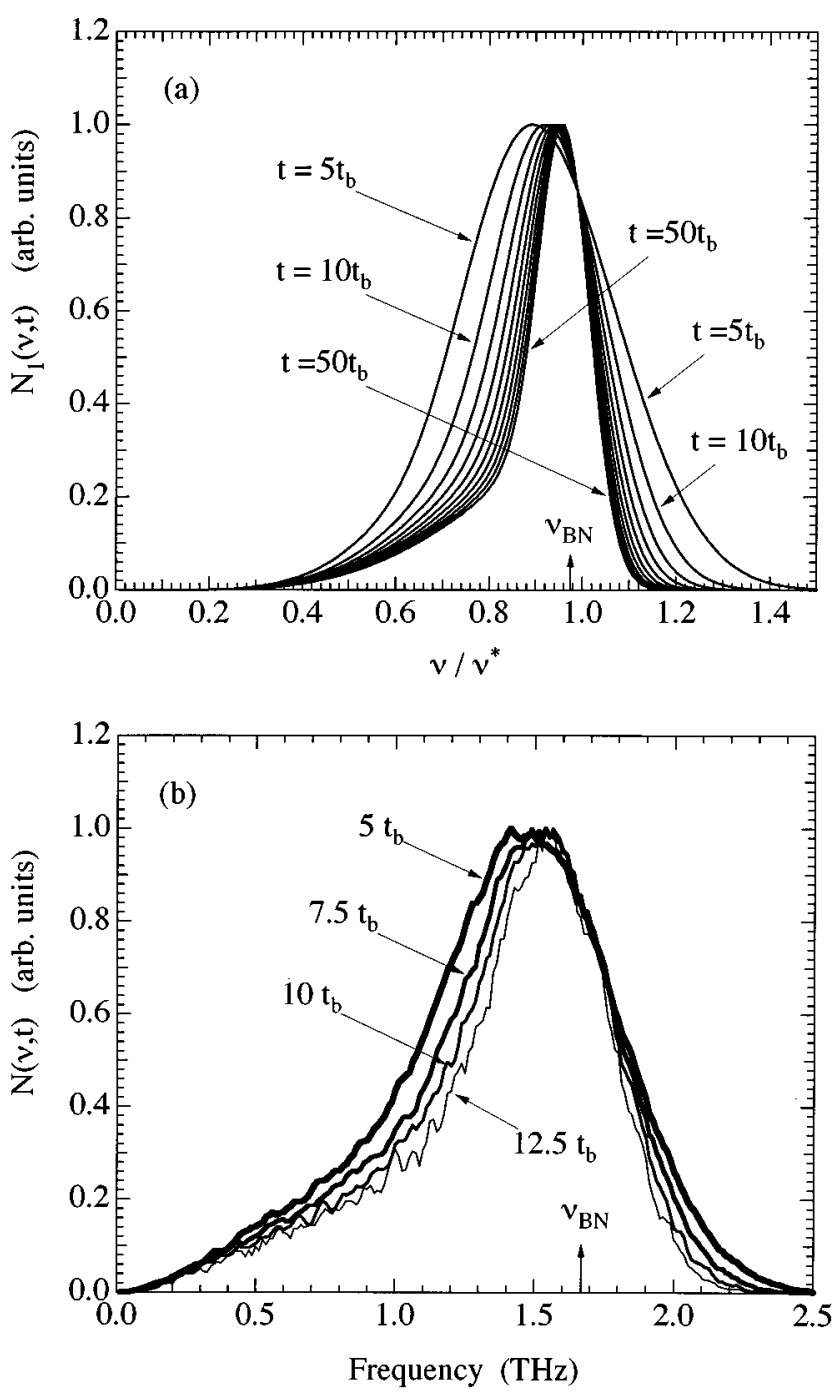

FIG. 2. Frequency dependences of phonon concentrations in the sample at several times $t$ much later than ballistic time of flight $t_{b}$. (a) The solutions $N_{1}$ of Eq. (3) and (b) the Monte Carlo results for $R=5.5 \mathrm{~mm}$. To compare the profiles the maxmum value of each trace is normalized to unity.

kept only the first eigenwave in the Fourier series putting $N(\nu, r, t)=N_{1}(\nu, t) \sin (\pi r / R) / r$. Now $N_{1}(\nu, t)$ obeys the integro-differential equation

$$
\left[\frac{\partial}{\partial t}+\frac{1}{\tau_{A}(\nu)}+\frac{1}{\tau_{D}(\nu)}\right] N_{1}(\nu, t)=\int_{\nu}^{\infty} d \nu^{\prime} N_{1}\left(\nu^{\prime}, t\right) \Gamma\left(\nu^{\prime}, \nu\right),
$$

with $\tau_{D}^{-1}=\pi^{2} D(\nu) / R^{2}$ for a sphere. This is the equation which Esipov analyzed closely. At a time $t$ much later than $t_{b}$ Esipov found that the solution $N_{1}$ behaves as $\sim \exp \left[-t / t_{0}(\nu)\right]$, the time constant $t_{0}$ being determined from

$$
\left[t_{0}(\nu)\right]^{-1}=\min _{\tilde{\nu} \geqslant \nu}\left[\frac{1}{\tau_{A}(\widetilde{\nu})}+\frac{1}{\tau_{D}(\widetilde{\nu})}\right] .
$$

This can be readily seen by discretizing the frequency $\nu$ as $\nu_{1}, \nu_{2}, \ldots, \nu_{n}, \ldots$, and converting Eq. (3) into a set of coupled linear differential equations for $\quad N_{1}\left(\nu_{1}, t\right), N_{1}\left(\nu_{2}, t\right), \ldots, N_{1}\left(\nu_{n}, t\right), \ldots, \quad$ where 

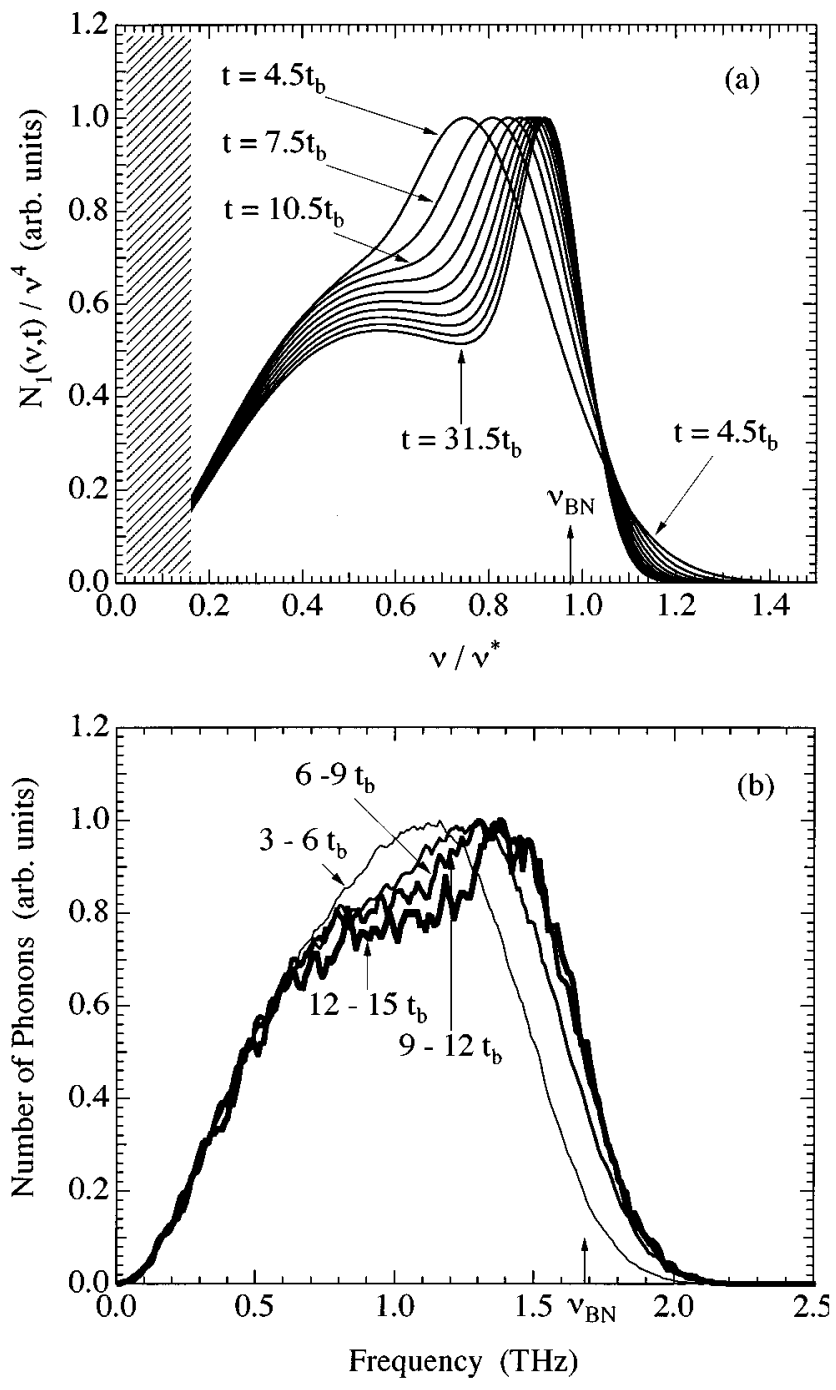

FIG. 3. Frequency dependences of the phonon numbers arriving at the surface of the spherical sample are plotted at several times $t$ much later than ballistic time of flight $t_{b}$. (a) The results derived from the solution of Eq. (3) and (b) the Monte Carlo results for $R=5.5 \mathrm{~mm}$ integrated over the time intervals indicated. The average arrival times of the traces in (b) correspond to the arrival times labeled for the first four traces of (a). To compare the profiles the maximum value of each trace is normalized to unity. The diffusion approximation fails at the low-frequency region hatched in (a).

$\nu_{1}<\nu_{2}<\ldots<\nu_{n}<\ldots$. Because $\tau_{A}^{-1}$ and $\tau_{D}^{-1}$ are proportional to $\nu^{5}$ and $\nu^{-4}$, respectively, their sum has a minimum at $\nu=\nu_{\mathrm{BN}} \equiv(4 / 5)^{1 / 9} \nu^{*}$, where

$$
\nu^{*} \equiv\left(\frac{\pi^{2} v^{2}}{3 R^{2} A B}\right)^{1 / 9}
$$

and the corresponding relaxation rate of the phonon signal at $t \gg t_{b}$ is

$$
\left[t_{0}\left(\nu_{\mathrm{BN}}\right)\right]^{-1}=\frac{9}{4} A \nu_{\mathrm{BN}}^{5} .
$$

Thus, if the initial frequency is high enough, the phonons with $\nu>\nu_{\mathrm{BN}}$ decay with a decay rate given by $1 / t_{0}(\nu)=1 / \tau_{A}(\nu)+1 / \tau_{D}(\nu)$, whereas those with $\nu \leqslant \nu_{\mathrm{BN}}$ de-

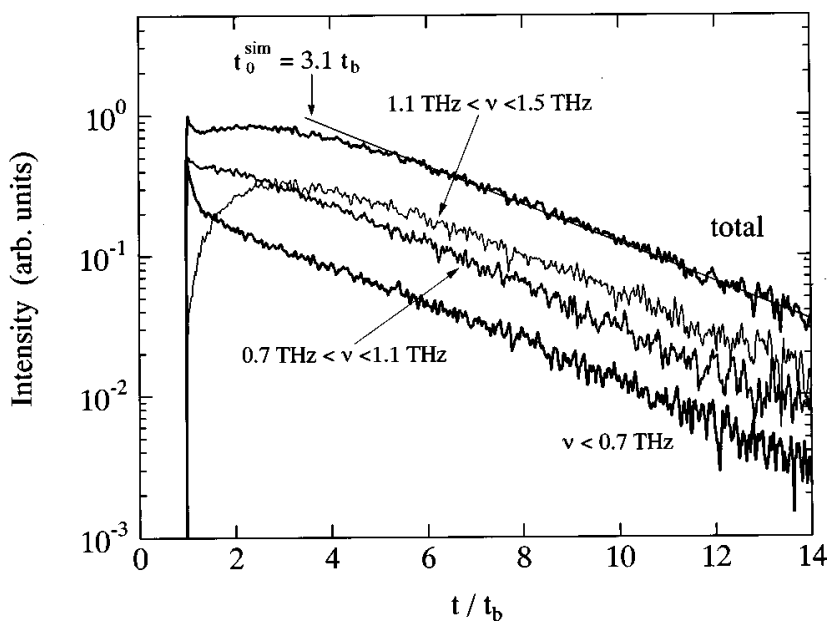

FIG. 4. Monte Carlo results for intensities vs arrival time for phonons with frequencies within the intervals indicated. The straight line indicates the exponentially decreasing profile with time constant $t_{0}^{\mathrm{sim}}=3.1 t_{b} . R=5.5 \mathrm{~mm}$ and $v=5.91 \times 10^{5} \mathrm{~cm} / \mathrm{s}$ are used.

cay with the same time constant $t_{0}\left(\nu_{\mathrm{BN}}\right)$. It should be noted that this bottleneck frequency $\nu_{\mathrm{BN}}$ depends on the system size but not on the initial frequency of phonons exited. Numerically $\nu_{\mathrm{BN}}=1.68 \mathrm{THz}$ and $t_{0}\left(\nu_{\mathrm{BN}}\right)=3.0 t_{b}$ for a spherical silicon sample of radius $R=5.5 \mathrm{~mm}$, where we have used $v=5.19 \times 10^{5} \mathrm{~cm} / \mathrm{s}$ for the Debye velocity and $A=1.2 \times 10^{-56} \mathrm{~s}^{4}$ for the mode-averaged decay constant in silicon.

In order to see more explicitly the existence of the bottleneck frequency we have solved Eq. (3) numerically and plotted the time evolution of the phonon concentration $N_{1}(\nu, t)$. Figure 2(a) exhibits the solutions of Eq. (3) for ten selected times much later than the ballistic time of flight $t_{b}$. The frequency distributions of phonons quasidiffusing in the sample exhibit sharp peaks which move towards $\nu=\nu_{\mathrm{BN}}$ for $t \rightarrow \infty$. This clearly indicates the existence of the predicted bottleneck frequency for phonons at $t \gg t_{b}$; that is, the relaxation time is the longest at $\nu=\nu_{\mathrm{BN}}$. The abrupt decrease of $N_{1}(\nu, t)$ for $\nu>\nu_{\mathrm{BN}}$ is due to the strong anharmonic decay as predicted. The phonons for $\nu \leqslant \nu_{\mathrm{BN}}$ should decay with the same time constant $t_{0}\left(\nu_{\mathrm{BN}}\right)$ but the shape of the $\nu$-dependent profile of $N_{1}(\nu, t)$ depends on the selected form of $P\left(\nu^{\prime}, \nu\right)$. It should be noted that the same relaxation rate for phonons of $\nu<\nu_{\mathrm{BN}}$ at $t \gg t_{b}$ arises from the fact that those phonons are produced via a decay of the phonon with $\nu=\nu_{\mathrm{BN}}$.

Figure 2(b) also plots the time evolutions of phonon concentration calculated by the Monte Carlo simulation in the same sample geometry. An initial frequency (4 THz) higher than $\nu_{\mathrm{BN}}$ is assumed. The simulated frequency distributions at later times also show peaks around $\nu_{\mathrm{BN}}$ and their profiles are very similar to $N_{1}(\nu, t)$ plotted in Fig. 2(a) except in the low-frequency region. Note that the low-frequency phonons propagate nearly ballistically and are excluded in the calculation with the diffusion approximation.

The phonon flux in the diffusion approximation is given by $\mathbf{J}(\nu, \mathbf{r}, t)=-D(\nu) \nabla N(\nu, \mathbf{r}, t)$ (Fick's law). So in this approximation the number of phonons hitting the surface of the sphere at $t \gg t_{b}$ should be proportional to $\left.D(\nu) \nabla_{r} N\right|_{r=R} \sim \nu^{-4} N_{1}(\nu, t)$. We have shown in Fig. 3 the frequency dependence of the phonon flux arriving at the sur- 
face of silicon sample. The majority of phonons detected at the sample surface consist of those with $\nu<\nu_{\mathrm{BN}}$. We note that the frequency dependence at late times exhibits a bump at about $\nu_{\mathrm{BN}} / 2$ in addition to the peak around $\nu_{\mathrm{BN}}$. This bump becomes more significant when we assume $P\left(\nu^{\prime}, \nu\right) \sim \nu^{n}\left(\nu^{\prime}-\nu\right)^{n} /\left(\nu^{\prime}\right)^{n+1}$ with $n>2$; that is, the anharmonic decay splits a parent phonon more frequently into daughter phonons of equal energy. It should be pointed out that the solution of the kinetic equation [Fig. 3(a)] coincides qualitatively with the Monte Carlo result [Fig. 3(b)] which also exhibits shoulders in addition to peaks near $\nu_{\mathrm{BN}}$ at later times. However, the diffusion approximation again leads to an unphysical (divergent) result at the low-frequency region [hatched region in Fig. 3(a)] where this approximation is no longer valid.

The Esipov prediction that the time constant $t_{0}$ is the same for any phonons with $\nu<\nu_{\mathrm{BN}}$ can be also confirmed by Monte Carlo calculations. This is done by plotting the time traces of the phonon intensity hitting the sample surface (Fig. 4). The initial frequency $\nu_{0}$ of the phonons excited is again 4 $\mathrm{THz}\left(>\nu_{\mathrm{BN}}\right)$, but the results are insensitive to the frequency assumed if $\nu_{0}$ higher than $4 \mathrm{THz}$ is chosen. We see that the tail of the simulated phonon signal exhibits an exponential decay in time, and the decay constants for three chosen frequency intervals $\nu<0.7 \mathrm{THz}, 0.7 \mathrm{THz}<\nu<1.1 \mathrm{THz}$, and $1.1 \mathrm{THz}<\nu<1.5 \mathrm{THz}$ are the same; that is, $t_{0}^{\mathrm{sim}}=3.1 t_{b}$. In addition this time constant is consistent with $t_{0}\left(\nu_{\mathrm{BN}}\right)=3.0 t_{b}$ given by Eq. (6) for $R=5.5 \mathrm{~mm}$, but slightly smaller than the experimental value $t_{0}^{\text {expt }}=3.6 t_{b}$ observed in the slab geometry. A more quantitative comparison with the experiments would require Monte Carlo simulation including three distinct phonon modes and the exact sample geometry of the experiments.

To conclude, we have solved numerically the kinetic equation for quasidiffusion and also conducted Monte Carlo calculations of phonon propagation in the presence of both elastic scattering and frequency downconversion. Both results have confirmed the existence of the bottleneck frequency $\nu_{\mathrm{BN}}$ predicted by Esipov, which governs the quasidiffusion of phonons at $t>t_{b}$ : (1) The frequency distribution of phonons in a sample has a sharp peak at $\nu=\nu_{\mathrm{BN}}$, and (2) the quasidiffusive tails of the phonons with $\nu<\nu_{\mathrm{BN}}$ decay with the same time constant $t_{0}\left(\nu_{\mathrm{BN}}\right)$. However, the kinetic equation we consider is based on the diffusion approximation and hence fails at low phonon frequencies. It would be an interesting challenge to construct a model which explains both the ballistic and quasidiffusive regimes including threephonon branches.

The authors would like to thank S. E. Esipov and H. J. Maris for valuable discussions, and O. B. Wright for useful comments on the manuscript. This work was supported in part by a Grant-in-Aid for Scientific Research from the Ministry of Education, Science and Culture of Japan (Grant No. 09640385).
${ }^{1}$ B. Taylor, H. Maris, and C. Elbaum, Phys. Rev. Lett. 23, 416 (1969); Phys. Rev. B 3, 1462 (1971).

${ }^{2}$ G. A. Northrop and J. P. Wolfe, in Nonequilibrium Phonon Dynamics, edited by W. E. Bron (Plenum, New York, 1985), p. 165.

${ }^{3}$ S. Tamura, Phys. Rev. B 27, 858 (1983); 30, 849 (1984).

${ }^{4}$ S. Tamura, Phys. Rev. B 31, 2574 (1985).

${ }^{5}$ S. Tamura and H. J. Maris, Phys. Rev. B 31, 2595 (1985).

${ }^{6}$ A. Berke, A. P. Mayer, and R. K. Wehner, J. Phys. C 21, 2305 (1988)

${ }^{7}$ D. V. Kazakovtsev and Y. B. Levinson, Phys. Status Solidi B 96, 117 (1979).

${ }^{8}$ Y. B. Levinson, in Nonequilibrium Phonons in Nonmetallic Crystals, edited by W. Eisenmenger and A. A. Kaplyanskii (NorthHolland, Amsterdam, 1986), p. 91.

${ }^{9}$ W. E. Bron, Y. B. Levinson, and J. M. O'Conor, Phys. Rev. Lett. 49, 209 (1982).

${ }^{10}$ T. E. Wilson, F. M. Lurie, and W. E. Bron, Phys. Rev. B 30, 6103 (1984)
${ }^{11}$ J. A. Shields, M. E. Msall, M. S. Carroll, and J. P. Wolfe, Phys. Rev. B 47, 12510 (1993).

${ }^{12}$ M. E. Msall, S. Tamura, S. E. Esipov, and J. P. Wolfe, Phys. Rev. Lett. 70, 3463 (1993).

${ }^{13}$ M. E. Msall and J. P. Wolfe, Phys. Rev. B 56, 9557 (1997).

${ }^{14}$ B. A. Young, B. Cabrera, and A. T. Lee, Phys. Rev. Lett. 64, 2795 (1990).

${ }^{15}$ H. J. Maris and S. Tamura, Phys. Rev. B 47, 727 (1993).

${ }^{16}$ H. J. Maris, J. Low Temp. Phys. 93, 355 (1993).

${ }^{17}$ S. E. Esipov, M. E. Msall, B. Cabrera, and J. P. Wolfe, J. Low Temp. Phys. 93, 377 (1993).

${ }^{18}$ A. T. Lee, B. Cabrera, B. L. Dougherty, M. J. Penn, J. G. Pronko, and S. Tamura, Phys. Rev. B 54, 3244 (1996).

${ }^{19}$ H. J. Maris, Phys. Rev. B 41, 9736 (1990).

${ }^{20}$ S. Tamura, Phys. Rev. B 48, 13502 (1993).

${ }^{21}$ S. Tamura, J. Low Temp. Phys. 93, 433 (1993).

${ }^{22}$ S. R. Esipov, Phys. Rev. B 49, 716 (1994). 